\title{
Sunspot Cycle Minima and Pandemics: The Case for Vigilance?
}

\section{Wickramasinghe NC ${ }^{1,2}$, Edward J Steele ${ }^{2,3}$, Wainwright $\mathbf{M}^{1,2,5}$, Gensuke Tokoro ${ }^{2,6}$, Manju Fernando ${ }^{2}$ and Jiangwen $\mathbf{Q u}^{4}$}

${ }^{1}$ Buckingham Centre for Astrobiology, University of Buckingham, UK

${ }^{2}$ Centre for Astrobiology, University of Ruhuna, Matara, Sri Lanka

${ }^{3} \mathrm{CY}$ O'Connor ERADE Village Foundation, Piara Waters, Australia

${ }^{4}$ Department of Infectious Disease Control, Tianjin Centers for Disease Control and Prevention, China

${ }^{5}$ Department of Molecular Biology and Biotechnology, University of Sheffield, Sheffield S10 2TN, UK

${ }^{6}$ Institute for the Study of Panspermia and Astroeconomics, Gifu, Japan

${ }^{*}$ Corresponding author: Wickramasinghe NC, Buckingham Centre for Astrobiology, University of Buckingham, Buckingham MK18 1EG, UK, Tel: +44 2920752146; Email: ncwick@gmail.com

Received date: June 7, 2017; Accepted date: June 20, 2017; Published date: June 23, 2017

Copyright: (C) 2017 Wickramasinghe NC, et al. This is an open-access article distributed under the terms of the Creative Commons Attribution License, which permits unrestricted use, distribution, and reproduction in any medium, provided the original author and source are credited.

\begin{abstract}
Direct records of sunspots and the solar cycle have been maintained in astronomical observatories for about $1610 \mathrm{AD}$, while indirect records derived from ${ }^{14} \mathrm{C}$ analysis of ice cores go back to about $900 \mathrm{AD}$. Minima in the sunspot cycle present conditions conducive to the entry or activation of new pathogens and also for mutations of already circulating bacteria and viruses. Three grand minima of solar activity on record-the Sporer minimum (1450-1550 AD), Maunder minimum (1650-1700 AD) and the Dalton minimum (1800-1830) have all been marked by a preponderance of pandemics-Small Pox, English Sweats, Plague and Cholera. The sunspot numbers recorded for the present period 2002-2017 include the deepest sunspot minimum (Cycle 23-24) since records began, and a trend to declining numbers throughout the cycle. The same period has seen the resurgence of several pandemicsSARS, MERS, Zika, Ebola, Influenza A. We consider it prudent to take note of these facts whilst planning future strategies for pandemic surveillance and control.
\end{abstract}

Keywords: Sunspot cycle; Panspermia; Pandemics

\section{Introduction}

The possibility of linking sunspots with pandemics was first suggested in 1977 by Hope-Simpson [1] who pointed out that many pandemics of influenza in history occurred close to times of sunspot maxima. Hoyle and Wickramasinghe [2-4] re-examined this proposal using a more extended dataset and concluded that although the coincidences were not precise, the two sets of data-influenza and sunspot-were phase-locked such that a causal link was likely. This work was recently extended by Qu [5] who found a more generalised result, namely that both certain and possible pandemics fall within \pm 2 years of sunspot extrema (maxima and minima).

Maxima in the sunspot cycle are characterised by high daily sunspot numbers, frequent solar flares, coronal discharges and X-ray emission. High fluxes of X-rays reach the Earth's upper atmosphere but are almost totally absorbed by the lower atmosphere. A more important property of a sunspot maximum is that the interplanetary magnetic field near the Earth remains high, being generated and maintained by the flow of electrons from the sun. As such the Earth would be effectively shielded from the ingress of charged dust grains as well as galactic cosmic ray protons.

It is to sunspot minima that we must turn to seek a possible explanation of the onset of pandemics. Sunspot minima are characterised by a weakening of the interplanetary magnetic field near the Earth, which allows for the entry of Galactic Cosmic Rays (GCRs) as well as electrically charged bacteria and viruses to the Earth. When GCRs collide with the atmosphere they produce a cascade of secondary particles including neutrons and muons that continue to penetrate the atmosphere. The cascade continues until the particle energy becomes too low and GCRs are effectively stopped. This happens at $16-20 \mathrm{~km}$. Significant neutron fluxes do, however, reach ground level and have the potential of causing mutations in both viruses and cells in general.

More significantly, in our view, during solar minimum, new viruses, bacteria and other microscopic biological entities can penetrate the interplanetary magnetic field barrier and reach the stratosphere. Descent of such particles to ground level by means of gravitational settling might take months or years depending on size. The final descent phase through the troposphere would be mostly controlled by meteorological events. It is also of interest to note that the first descent of viral-sized particles deposited in the stratosphere will occur at places where the stratosphere is thinnest; and by this argument populated areas of China lying eastward of the Himalayan mountain range would present the best candidates. It is therefore not surprising to find that first strikes of new or renewed viral diseases are often recorded in China. We should however stress that not every minimum in the sunspot cycle would be associated with a new epidemic or new pathogen. Additional conditions need to be fulfilled, most importantly that the Earth has recently encountered a stream of cometary debris containing disease-causing dust-e.g. phages, virions, microorganisms.

Hoyle and Wickramasinghe [2] argued that the recurrent patterns of certain pandemics throughout history exhibiting long periods of dormancy might be best understood by extinctions followed by reintroductions, possibly involving a long period comet. With the theory of cosmically interconnected biology coming to the fore such a possibility has currently acquired a renewed relevance. 


\section{Sunspot Minima and Plagues}

In addition to the oscillation of sunspot numbers with a 11-year period, several "grand minima" where low sunspot numbers persist over several cycles, are known to have occurred (Figures 1a and 1b) [6,7].

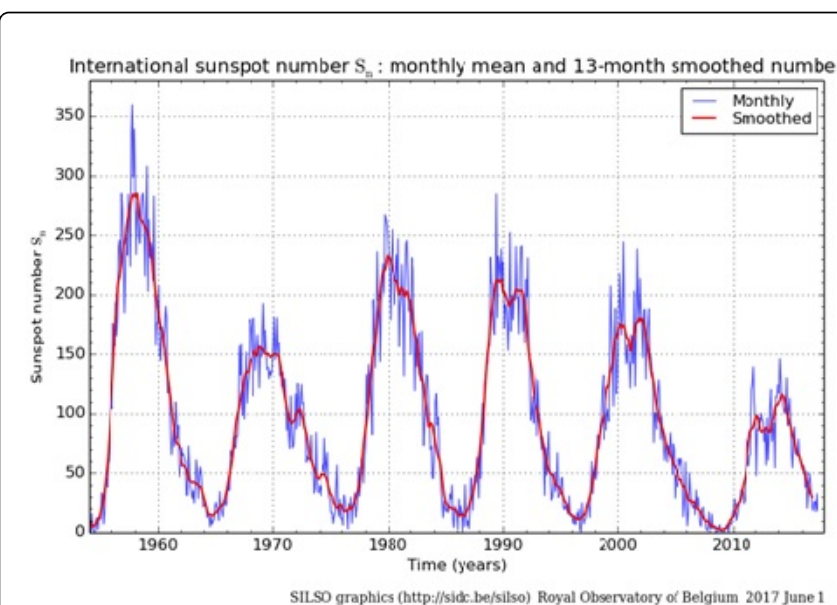

Figure 1a: Monthly sunspot numbers from 1954 to the present which shows the 11 year sunspot cycle and a protracted minimum followed by a low maximum 2014. The blue curve is the monthly mean, and the red curve is the 13-month smoothed average. Data is from public access records of the Royal Observatory of Belgium.

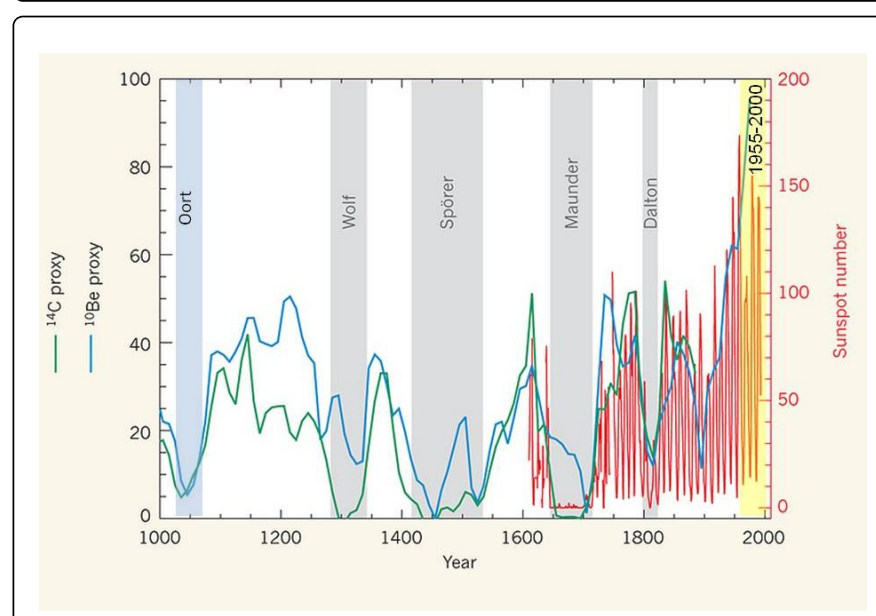

Figure 1b: The graph adapted from Charbonneau [7] shows the 11year cyclical variation in the number of sunspots since records began (red curve) and two equivalents are based on the production rate of the radioactive nuclides carbon $-14\left({ }^{14} \mathrm{C}\right)$ and beryllium-10 $\left({ }^{10} \mathrm{Be}\right)$ resulting from the addition of neutrons from galactic cosmic rays which is at a maximum during sunspot minima. The Oort, Wolf, Spörer, Maunder and Dalton minima are clearly visible in this record. The yellow highlighted region is that in Figure 1a.

The Maunder minimum from 1645-1715 was marked by an almost total absence of sunspots for many days, and the Dalton minimum from 1790-1830 witnessed a significant reduction of sunspot numbers over several cycles. An earlier period of reduced activity (1400-1520) is known as the Sporer minimum has recently been confirmed indirectly in the "proxy" record of ${ }^{14} \mathrm{C}$ and ${ }^{10} \mathrm{Be}$. High fluxes of neutrons coming from the decay of cosmic ray protons during sunspot minima cause the production of ${ }^{14} \mathrm{C}$ and ${ }^{10} \mathrm{Be}$ which is deposited in ice cores.

Figure $1 \mathrm{~b}$ shows the 11-year cyclical variation in the number of sunspots since records began (red curve) combined with two equivalents are based on the production rate of the radioactive nuclides ${ }^{14} \mathrm{C}$ ) and ${ }^{10} \mathrm{Be}$. The Wolf, Spörer, Maunder and Dalton minima are all clearly visible in this record.

All three major sunspot minima in Figure 1b-Sporer, Maunder and Dalton are known to have been associated with devastating pandemics caused by viral or bacterial agents. Whilst some of these agents could be interpreted as being newly introduced to the Earth, others probably represented mutated or re-activated entities that were in circulation at the time.

The "English Sweats" that continued through most of the period of the Sporer minimum still remains an unsolved mystery for medical historians. Holinshed's Chronicles, published in 1557, described English sweating sickness as "so sharp and deadly that the "lyke was never hearde of to any manne's remembrance before that tyme." The disease is said to have begun abruptly with fever, severe body aches, abdominal pain, vomiting and profuse sweating, and death came swiftly. It has been speculated that a hitherto unknown virus may have been involved [8]. This is in contrast to bubonic plague in the Maunder Minimum, as well as cholera, and smallpox that are caused by several resident and circulating pathogens that gain pandemic-potential intermittently due to mutation or recombination events which presumably involve the acquisition of new genes (plasmids, phages, virions). It is also possible for an epidemic to be triggered when the microflora eco-balance in the upper respiratory tract is disrupted by a new pathogenic invader, thus causing a resident suppressed microorganism to replicate and flourish causing overt disease.

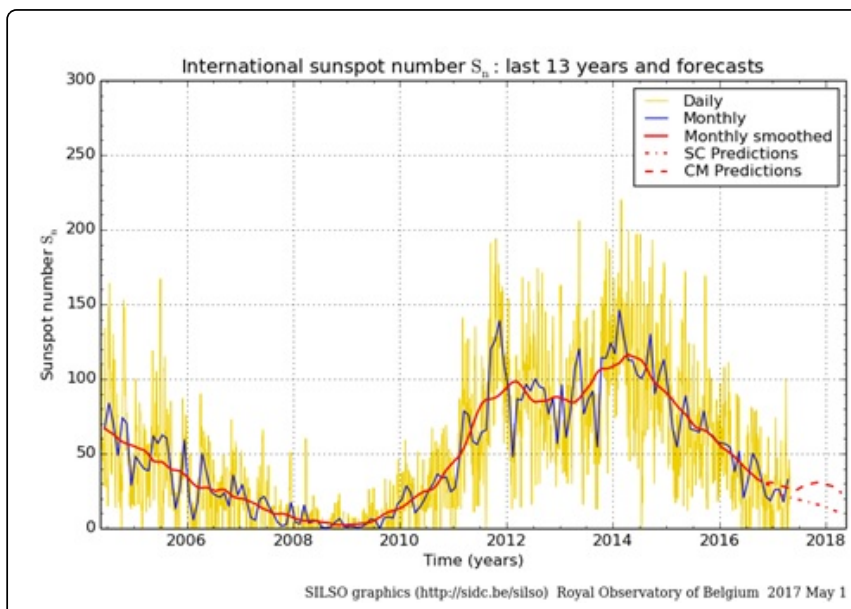

Figure 2: Sunspot data from 2004 to the 2017 from database of the Royal Observatory of Belgium. In the legend SC and CM refers to model predictions not relevant to the present study. Yellow curve shows daily sunspot numbers showing many days of zero spots between 2006 and 2011.

\section{A Modern Sunspot Minimum 2002-2015}

The modern period 2002-2017 has been characterised by the lowest solar activity since the Dalton minimum (Figure 1a). In particular the 
solar minimum between cycles 23 and 24 was exceptionally protracted with many days of almost no sunspots (Figure 2).

It was also marked by the lowest recorded values of the interplanetary magnetic field near the Earth, the lowest in 50 years since space measurements began [9]. These conditions taken together arguably provide the best opportunity for the onset of pandemics. An open gateway is offered for the ingress of new pathogens which are inevitably electrically charged in space. Virions, phages, bacteria, and other micron-sized biological entities can cross the weakened interplanetary magnetic field and readily reach the Earth's surface. A low or zero magnetic fields would also provide an opportunity for mutational events caused by increased fluxes of galactic cosmic rays (Table 1) [10].

\begin{tabular}{|l|l|}
\hline 2010-2016 & $\begin{array}{l}\text { Scarlet fever (Streptococcus } \\
\text { pyogenes) }\end{array}$ \\
\hline 2015 & Zika \\
\hline 2014 & Ebola \\
\hline 2013 & Influenza A H7N9 \\
\hline 2012 & MERS \\
\hline 2009 & Influenza H1N1(China, India, Sri Lanka) \\
\hline 2002 & SARS \\
\hline & \\
\hline
\end{tabular}

Table 1: Lists of conspicuous pandemic and epidemic events during the period 2002-2015.

An epidemic of particular interest is Scarlet Fever (SF) which has flared up in 2015-15 after several decades of relative dormancy. This is a disease caused by the bacterium Streptococcus pyogenes and has an antiquity that goes probably goes back to Hippocrates in the 4th century BC. Judged from the record of historical data in the Christian era the disease (judged by symptoms alone) appears to have had several exits and entrances with long interludes of remission. The disease emerged as a major scourge at the beginning of the Victorian era. From 1840-1883 SF was probably the most common childhood infection leading to high fatality rates in cities throughout Europe and the USA. In England two of Charles Darwin's children are known to have succumbed to the disease. An oscillatory pattern of occurrence of SF is recorded in death-rates per 100,000 in Boston for the period 1840-1940 [11].

The incidence rates of SF began to drop dramatically from the 1930's onwards and particularly after the introduction of antibiotics in the 1950 's. Although factors such as herd immunity are widely suggested for the long-term oscillations of incidence an alternative explanation would be the episodic variability of the genome of the causative bacterial population Streptococcus pyogenes. S. pyogenes DNA was amplified by Chalker et al. [12] with primers specific to particular emm genes and a spread of emm types were revealed in several districts of the UK. The sudden increase in virulence (from 2011 onward) was clearly not linked to emm type of the $S$. pyogenesbacillus, although in some investigations a dominance of emm99 seems to have been recorded in the UK [13].

Other epidemics or pandemics of viral diseases that were initiated during this period may also have shared a similar cause. SARS-CoV virus first flared up in China in 2002. A decade later in 2012 an outbreak of MERS-CoV started in the Middle East and spread sporadically throughout the world.

The Influenza subtype H1N1, which was involved in both the $1918 / 1919$ pandemic and the epidemics in $1976 / 1977$, reappeared in 2009 in India, China and elsewhere. In 2017 it was raging across Sri Lanka and neighbouring countries. In 2013 the Influenza subtype H7N9 appeared first in birds and later spread globally.

In 2015 the Zika virus which had been endemic since the 1950's acquired new characteristics including the capacity to cause microcephaly. Recently a gene mutation that caused the changed character of this virus has been identified by Liu et al. [14]. In 2014 the Ebola virus which was first recognised in Zaire, W. Africa in 1976 resurfaced to cause localised epidemics.

\section{Prediction and Mitigation Strategies}

From past records of the correlation of the sunspot cycle (prolonged minima) and pandemics it is clear that the onset of a deep minimum is a signal of action. We have stated elsewhere that the sunspot cycle could be a guide for closer scrutiny of circulating viruses, and monitoring their genetic variations. Equally important in our view is to monitor the stratosphere for the presence of viral entities that may pose a pandemic threat if and when they reach ground level. Early balloon collections of stratospheric aerosols [15-17] need to be refined and more funds devoted to this project if a future pandemic can be avoided. At sea level we are also proposing to carry out a regular sampling of ocean water in Sri Lanka to detect the arrival of new viruses. Some 10 million viruses have been identified in a single drop of ocean water at any moment, and to keep a track of their evolution over time would, in our view, be extremely important. A Virus Guard Project, similar to the Space Guard Project proposed by Sir Arthur Clarke for detecting asteroids and comets, may be needed to protect us. Sir Arthur Clarke remarked that the dinosaurs became extinct because they failed to initiate a Space Guard Project. Let us not default in our duty by future generations of humans to start such a Virus Guard Project.

\section{References}

1. Hope-Simpson RE (1978) Sunspots and flu: a correlation. Nature 275: 86.

2. Hoyle F, Wickramasinghe C (1979) Diseases from Space London.Dent.

3. Hoyle F, Wickramasinghe NC (1990) Sunspots and influenza. Nature 343: 304.

4. Hoyle F, Wickramasinghe NC (1990) Influenza-evidence against contagion: discussion paper. JRSM 83: 258.

5. Qu J (2016) Is sunspot activity a factor in influenza pandemics? Rev Med Virol 26: 309.

6. Usoskin IG (2017) A history of solar activity over millennia. Living Rev Sol Phys 14: 3.

7. Charbonneau P (2013) Solar physics: The planetary hypothesis revived. Nature 493: 613-614

8. Heyman P, Simons L, Cochez C (2014) Were the English sweating sickness and the Picardy sweat caused by hantaviruses? Viruses 6: 151-171.

9. de Toma G, Gibson SE, Emery BA, Arge CN (2010) The Minimum between Cycle 23 and 24: Is Sunspot Number the Whole Story? InSOHO-23: Understanding a Peculiar Solar Minimum 428: 217.

10. Qu J, Gao Z, Zhang Y, Wainwright M, Omairi T (2016) Sunspot activity, influenza and ebola outbreak connection. J Astrobiol Outreach 31: 1-7.

11. Krause RM (2002) Evolving microbes and re-emerging streptococcal disease. Clin Lab Med 22: 835-48. 
Citation: Wickramasinghe NC, Steele E, Wainwright M, Tokoro G, Fernando M, et al. (2017) Sunspot Cycle Minima and Pandemics: The Case for Vigilance? Astrobiol Outreach 5: 159. doi:10.4172/2332-2519.1000159

Page 4 of 4

12. Chalker V, Jironkin A, Coelho J, Al-Shahib A, Platt S, et al. (2014) Genome analysis following a national increase in Scarlet Fever in England. BMC Genomics 10: 18-224.

13. Turner CE, Abbott J, Lamagni T, Holden MT, David S, et al. (2015) Emergence of a new highly successful acapsular group a Streptococcus Clade of genotype emm89 in the United Kingdom. mBio 6: 4.

14. Liu Y, Liu J, Du S, Shan C, Nie K, et al. (2017) Evolutionary enhancement of Zika virus infectivity in Aedes aegypti mosquitoes. Nature 545: 482-486.
15. Harris MJ, Wickramasinghe NC, Lloyd D, Narlikar JV, Rajaratnam P, et al. (2002) Detection of living cells in stratospheric samples. InInternational Symposium on Optical Science and Technology 192-198. SPIE (International Society for Optics and Photonics).

16. Wainwright M, Omairi $\mathrm{T}$ (2016) New evidence that life is currently arriving to Earth from space. J Astrobiol Outreach 4: 1-7.

17. Wainwright M, Rose CE, Baker AJ, Wickramasinghe NC, Omairi T, et al. (2015) Biological entities isolated from two stratosphere launchescontinued evidence for a space origin. J Astrobiol Outreach 3: 1-5. 